\title{
Tracheal Aspirate Cytology of 43 High-Risk Neonates to Predict Bronchopulmonary Dysplasia Early
}

\author{
Sahar Ali Mohamed", Hossam Eldin Mohammed \\ Department of Pathology, Al Galaa Teaching Hospital, General Organization of Teaching Hospitals and Institutes (GOTHI), Cairo, Egypt \\ Email address: \\ Saharalimohamed.65@hotmail.com(S.A. Mohamed),hossammohammed@yahoo.com(H. E. Mohamed) \\ ${ }^{*}$ Corresponding author

\section{To cite this article:} \\ Sahar Ali Mohamed, Hossam Eldin Mohammed. Tracheal Aspirate Cytology of 43 High-Risk Neonates to Predict Bronchopulmonary \\ Dysplasia Early. American Journal of Laboratory Medicine. Vol. 4, No. 3, 2019, pp. 65-69. doi: 10.11648/j.ajlm.20190403.13
}

Received: June 19, 2019; Accepted: July 1, 2019; Published: July 12, 2019

\begin{abstract}
The main goal of this study was to assess the cytological evaluation of tracheal aspirate, in order to predict the early diagnosis of bronchopulmonary dysplasia (BPD) in high-risk babies during mechanical ventilation. Method: Samples from 43 infants (aged $<34$ weeks and weighed $\leq 1500 \mathrm{~g}$ ) were examined cytologically; who were admitted to Pediatric Intensive Care Units and diagnosed as respiratory distress syndrome by clinical and radiological criteria. Results: Up to 150 tracheal aspirates from 43 infants were examined to demonstrate cytological changes in bronchial epithelial cells that progressed to dysplasia. It was cytologically designated Class III. According to cytological criteria, all intubated infants had reactive hyperplastic and metaplastic epithelial changes. There were dysplastic changes in 23 out of 33 who developed BPD and 7 out of 40 who had a normal outcome. The variation between the numbers of neonates that had dysplasia in the BPD and the non-BPD group was significant $(\mathrm{P}<0.002$ by chi-square test). Conclusion: In order to monitor the development of lung injury and repair, tracheal aspirate cytology is simple, non-invasive and fast. Cytological monitoring of respiratory distress infants may be helpful in the early diagnosis and prognosis of the developing BPD. Evaluating new BPD preventive treatment schemes may also be valuable.
\end{abstract}

Keywords: Bronchopulmonary Dysplasia (BPD), Cytology, Microscopic, Neonates, Predict, Tracheal Aspirate

\section{Introduction}

Bronchopulmonary Dysplasia (BPD) is a severe chronic lung disease that affects newborns precipitately due to lung vascular and alveolar development failure [1, 2]. These infants may be hospitalized, accustomed to extra oxygen or a ventilator while having long-lasting airway obstruction symptoms [3, 4].

BPD clinical definition is the persistent oxygen requirement at a postmenstrual age of 36 weeks with reliable radiological changes limited to oxygen requirements at 28 days $[5,6]$. Its phenotype results from a complex process in which both antenatal and postnatal factors are predictors for BPD [7, 8].

Currently, there are no accessible viable biomarkers to predict BPD. In addition, clinical or pharmacological treatments are needed to prevent or reverse BPD. These challenges remain a critically important study area for BPD
[9, 10]. BPD also has far-reaching adverse financial and health effects, including increased use of pediatric care, increased hospitalizations, and poorer early childhood neurodevelopmental and cognitive outcomes $[11,12]$.

The purpose of this study is to consider ways to determine the relationship of cytological examination of tracheal aspirate in high-risk neonates, who suspect to develop BPD, whether the procedure of tracheal lavage and aspiration in newborn children with endotracheal tubes was routinely performed for the pulmonary toilet.

\section{Material and Method}

\subsection{Patients}

Forty-three premature infants, admitted from 2011 to 2017 to Neonatal Intensive Care Units in various hospitals, suffered from respiratory distress. The study excluded children with neuromuscular disease, congenital anomalies, 
or pulmonary hemorrhage. Our study limited the population; almost all of them had the same radiological manifestation and clinical details. Infants $<34$ weeks gestational age, weighed $\leq 1500 \mathrm{~g}$, and received mechanical ventilation on the first day of life, were eligible. The protocol was approved by the Human Ethics Committee for research.

\subsection{Tracheal Aspirates}

The tracheal aspirate was collected during routine endotracheal tube toilet on days 1, 3, 5, 7 and then twice weekly on a regular basis until extubation from all infants born at 28 weeks gestation maternity in keeping with Todd et al. [12].

One hundred and fifty samples were collected where specimen ranged from 1 to 5 and assessed accordingly. A sterile suction catheter was passed to the end of the endotracheal tube and suction was applied while the catheter was withdrawn. The tracheal aspirates used were undiluted. The aspirates were transferred onto a slide by squeezing the catheter, air dried, methanol fixed, stained with H\&E and examined by light microscope.

\subsection{Cytological Evaluation}

According to Merritt et al. cytological scoring system, cytological classes emerged:

1. Class I ( 1 to 4 days): associated with cohesive columnar cells exfoliation and small organized sheets of basically located tracheobronchial cells, the cytoplasm varied from deep basophilic to eosinophilic depending on its preservation and regular oval nucleus, with low nuclear/cytoplasmic ratio [13]. (Figure 1-A)

2. Class II (4 to 10 days): during which tracheobronchial cell regeneration was prominent and there were reactive cells, characterized by hyperplastic characteristics. Loss of cellular arrangements, oval irregular, and was often characterized by three dimensions. Crowded, overlapping, enlarged and irregular nuclei with finely granular chromatin and observed nuclei were major diagnostic characteristics.

The transition from the proliferative stage with gradual metaplasia was designated as Class II-III, where exist syncytial metaplastic polygonal squamous epithelial cells with central irregular nuclei, prominent nuclei in discrete dense eosinophilic cytoplasm. There was a high nuclear/cytoplasmic ratio. These characteristics were referred to as immature metaplastic cells as seen in sputum or cervical smear. (Figure 1-B) (Figure 1-B)

3. Class III (after 10 days): showed not only metaplasia but also nuclear characteristics of dysplasia. The nuclear/cytoplasmic ratio was high. The nuclei were hyperchromatic with coarse chromatin clumped and infrequent visible nuclei. The background was amorphous, showing inspissated casts, bacteria, blood, and inflammatory cell infiltration. (Figure 1-C)

\subsection{Clinical Care}

All newborn children have been treated according to approved protocols of the Neonatal Intensive Care Unit. Since many infants needed mechanical ventilation for some days, consecutive specimens could not be collected over a period of days or weeks.

\subsection{Statistical Analysis}

The collected data will be tabulated and analyzed using SPSS program version 20.0. The Chi-square test was used to compare variation between the BPD group and the non-BPD group. The results are considered statistically significant at a probability $p$ value of $<0.05$.

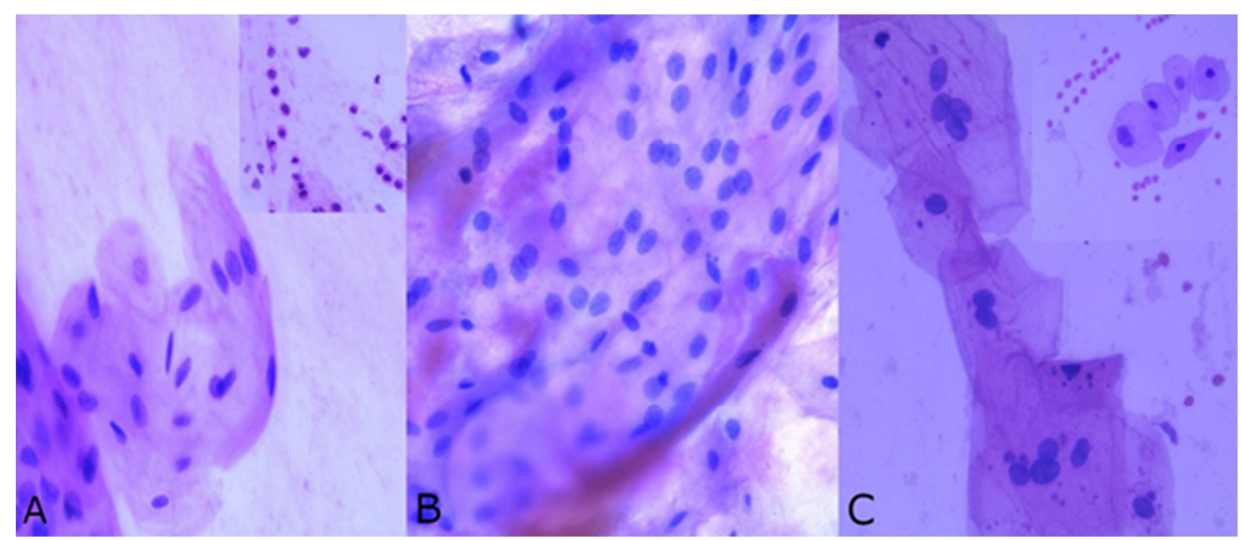

Figure 1. (A) Class I: Bronchial epithelial cells showing focal regular arrangement, early hyperplastic changes. (B) Class II: Hyperplastic bronchial epithelial cells with total disorganization of pattern, metaplastic bronchial epithelial cells, all demonstrating centrally located nuclei with a fine granular chromatin pattern and prominent nucleoli. (C) Class III: Dysplastic metaplastic cells with centrally located hyperchromatic irregular nuclei with coarsely granular chromatin and some in a syncytial arrangement, others lying singly (upper right corner), pattern. (A-C, H\&E stain, $x$, 400).

\section{Results}

This study enrolled 43 neonates, 3 cases died before reaching 37 weeks post-conceptual age within the first 2 weeks. Twenty three (57.5\%) babies had the same clinical characteristics and were diagnosed with long-term oxygen requirements (actual $\mathrm{FiO} 2>0.4$ ) and had radiological features to develop BPD in the first month. Of the 23 babies, 13 were males $(56.5 \%)$ and 10 were females $(43.5 \%)$. 
Table 1. BPD Patients were divided by gestational age.

\begin{tabular}{lll}
\hline Gestational age & Number & Percentage \\
\hline $33-35$ weeks & 11 & $48 \%$ \\
$31-33$ weeks & 6 & $26 \%$ \\
$29-31$ weeks & 3 & $13 \%$ \\
$27-29$ weeks & 2 & $9 \%$ \\
Less than 27 weeks & 1 & $4 \%$ \\
\hline
\end{tabular}

Table 2. The clinical data of patients at the time of admission and development of BPD.

\begin{tabular}{llllll}
\hline Gestational age & At admission & At initiation & $\mathbf{1}^{\text {st }}$ week & $\mathbf{2}^{\text {nd }}$ week & $\mathbf{3}^{\text {rd }}$ week \\
\hline $33-35$ weeks & None & None & None & 1 & 10 \\
$31-33$ weeks & None & None & 1 & 4 & 1 \\
$29-31$ weeks & None & None & None & 3 & \\
$27-29$ weeks & None & None & None & None & 1 \\
Less than 27 weeks & None & None & None & 10 & 12 \\
Total & None & None & 1 & \\
\hline
\end{tabular}

Only $1(4 \%)$ of 23 patients had cytological changes in developed BPD in the first week, $10(44 \%)$ patients in the second week, and the remaining $12(52 \%)$ patients in the third week. (Table 1)

Compared to the 7 (17.5\% of all neonates) patients who did not develop BPD and were discharged at different times, the $23(57.5 \%)$ patient who developed BPD was diagnosed with tracheal aspirate earlier. (Table 3)

In our study, a total of 150 tracheal aspirates was evaluated cytologically from all infants. A smear of inadequacy characterized by the absence of epithelial cells.

All infants, both in BPD and normal outcome, showed a cytological change from normal (Class I) to at least the metaplastic stage (Class II-III) through the hyperplastic stage. Of the 23 infants with BPD, 21 developed into a dysplastic (Class III) stage, with only two remaining in a hyperplasticmetaplastic (Class II-III) stage of ventilation up to 8 weeks after extubation.

Table 3. The relation between cytopathologic Classes and development of BPD.

\begin{tabular}{llll}
\hline Class & No. of patients (40) & \% & P value \\
\hline Class I (1-4 days) & 7 & 17.5 & NS \\
Class II (4-10 days) & 10 & 25 & NS \\
Class III (more than 10 days) & 23 & 57.5 & $<0.002$ \\
\hline
\end{tabular}

All infants with BPD generally showed hyperplasia within the first 2 days and by day 3 to 4 , they showed metaplastic epithelial changes. Dysplasia was noticeable in days 8 to 10 and did not regress for the full -time of ventilation in all 23 infants who were developing dysplasia just at the time of extubation.

In a normal-outcome group, all $33(82.5 \%)$ neonates carried on metaplasia (Class II-III) and 23 (57.5\%) upgraded to dysplasia (Class III). Hyperplasia frequently peaks at day 5 in these neonates and metaplasia gets noticed between days 5 and 8 . Completely dysplastic features only occurred on days 9 and 10 .

As BPD neonates, no regressive changes have been distinguished within the period of our study. There was significant variation in the number of neonates with dysplasia in the BPD group and non-BPD group $(\mathrm{P}<0.002)$. (Table 3$)$

\section{Discussion}

Mortality and BPD often require mechanical ventilation in premature neonates. Factors affecting BPD progress include impairment of normal development of the lung in association with lung damage and repair. [14]

Despite advances in the care of highly premature newborn children, BPD continues to remain a major open healthcare issue. Right now, our understanding of the BPD's precise pathogenesis is limited, which prevents the advancement of a chronic debilitating disease-focused treatment [1].

The main device within BPD's distinguishing evidence and observation, the chest $\mathrm{x}$-ray, appeared to have specific restrictions. Histologic interpretation with radiographic findings of BPD in 142 autopsied infants have shown a clear tendency for the pathologic extent of disease in term of fibrosis, interstitial and peribronchial smooth muscle proliferation, alveolar infiltrates, emphysematous foci, bronchiolar mucosal hyperplasia or dysplasia, and lymphatic dilatation to be more marked and severe than that appreciated radiographically, even allowing for the time lag between radiograph and death [13].

In the past, endotracheal aspiration cytology of respiratory epithelial cells was used to study pulmonary changes in BPD and it appeared to be a major indicator of pulmonary changes. In these studies, the newborn children were between 26 and 40 weeks gestation, weighed between 610 and $4000 \mathrm{gm}$ and were examined when BPD signs were more extreme [15-17, 19]

Doshi et al. correlated their cytological findings with histopathological findings and could only analyze and anticipate the results of disease in a large number of cases [18]. Todd et al. concluded that in their endotracheal aspirates, premature infants who had been intubated and ventilated 
mechanically had epithelial dysplasia [12].

Rothberg et al suggested that sequential analysis of tracheal aspirate cytology could allow one to predict, diagnose, and monitor BPD progression! Therefore, evidence of developing BPD would focus on more aggressive management of the controllable factors involved in BPD genesis [20]. So, the present study evaluated the predictive role of tracheal aspirate cytology in early detection and attempts to control BPD progression.

In our study, the cytological results found that 33 of 40 neonates on oxygen therapy, developed hyperplastic and metaplastic respiratory epithelial changes. Twenty-three of thirty-three progressed to frankly dysplastic cellular changes. These data are nearly matching with Rothberg et al who found all 39 studied neonates under oxygen therapy developed hyperplastic and metaplastic respiratory epithelial changes and 28 of 39 progressed to frankly dysplastic cellular alterations [20].

These results contradict Merritt et al. data in which all patients progressed to Class III cytology developed BPD [13, 15]. However, changes to the classification of Merritt et al. used in this current study can not explain the conflicting results obtained. The aspirates of Class III include metaplastic cells without any indication or distinction from superimposed dysplastic features. But in Todd et al. Class III was saved for those samples where true cellular dysplasia dominated, and indeed this more advanced cytological feature did not consistently predict the development of BPD [12].

Alternatively, the present study supports the results of D'Ablang and co-workers, who, despite an overall trend being evident, could not correlate cytological findings with long-term sequelae in individual cases [16].

In differentiating between cell size, shape and the nuclear/cytoplasmic ratio of metaplasia and dysplasia, Rothberg et al. showed no great significance. The main distinction was entirely dependent on the pattern of chromatin that was hyperchromatic and coarsely granular in dysplasia. They often observed that the nucleoli were sometimes visible within the pattern of chromatin. So they considered that the grading to Class III was reserved, showing not only metaplasia but also nuclear characteristics of dysplasia [13].

Merritt et al. noted a generally deliberate progression of time with changes in Class I occurring between days 1 and 4, Class II occurring from days 4 to 10 , and Class III occurring only after 10 days $[13,15]$. The results of the evaluation are not correlated with these evaluation times and are more in line with those of $\mathrm{D}^{\prime}$ Ablang et al. who noticed that cytological progression chronology was incidental individually, but faster than expected from histopathological studies [16].

The BPD group as a whole, achieved a dominant metaplastic exfoliation on days 5 and 8 in the present study, a delay of 48 hours before this effect was demonstrated by the normal-outcome group. Approximately, on days 7 and 8, the BPD group performed a predominant dysplastic pattern, the normal-outcome group again appreciated a 48 -hour check

and only on days 9 and 10 showed this pattern.

Our results were similar with Rothberg et al who noticed that on Day 3 and 4 all BPD groups had metaplastic features, a full 48 hours before the normal outcome group and dysplastic changes on Days 7 and 8, the normal outcome group experiencing a delay of 48 hours and displayed these changes only on Day 9 to 10 [20].

We found significant variation $(\mathrm{P}<0.002)$ between the numbers of neonates with dysplasia in the BPD and the nonBPD group who's had no regressive changes, which were distinguished within the duration of our study. (Table 3).

Similarly, with Rothberg et al, the distinction between the BPD number of neonates with dysplasia and the nonBPD group was significant (14 out of 15 vs. 14 out of 24; P $<0.02$ ). Over 82.5 percent of neonates carried on metaplasia (Class II-III) and 57.5 percent that upgraded to dysplasia (Class III) were found in the present study. Whereas, according to Rothberg et al's cytological scoring system, Class I was 17.5 percent of all considered neonates [20].

These results in agreement with Zhekova et al who concluded that the rate of metaplastic respiratory cells in TLF has an early predictive diagnostic value for CLD in high-risk neonates, metaplasia can be recognized as transitional in less than 40 percent of epithelial cells as it is variable; metaplasia between 40 percent and 80 percent of cells is associated with a hazard of broncho-obstructive syndrome in early infancy. Squamous metaplasia of greater than $80 \%$ of the cells in TLF has a diagnostic significant for CLD [21].

\section{Conclusion}

Tracheal aspirate cytology is simple, low-cost, noninvasive, easy and effective in early detection of BPD. This classification of tracheobronchial cytology should be beneficial in identifying and treating high-risk BPD neonates. It may also give a more coordinated implication for evaluating new therapy to prevent or adjust this disease. Lastly, we asked in this study whether the development of Class III cytological features can be prevented by improving clinical and pharmacological management in ventilated very low birth-weight infants. Assessing new BPD preventive treatment regimens may also be valuable. It may also be worthwhile.

All infants with BPD generally showed hyperplasia within the first 2 days and by day 3 to 4 , they showed metaplastic epithelial changes. Dysplasia was noticeable in days 8 to 10 and did not regress for the full -time of ventilation in all 23 infants who were developing dysplasia just at the time of extubation.

\section{Abbreviations Used}

BPD

FIO2

CLD

TFL

NS

\author{
Bronchopulmonary dysplasia \\ Fraction of inspired oxygen \\ chronic lung disease \\ tracheal lavage fluid \\ non significant
}




\section{Conflicts of Interest and Sources of Funding}

There are no conflicts of interest to confirm and also no sources of funding.

\section{Acknowledgements}

The authors gratefully acknowledge all staff members of the Neontal Intensive Care Units in the collection of the endotracheal aspirates as well as that of the Department of pathology at Al Galaa Teaching Hospital.

\section{References}

[1] LaGrandeur RG, Singhal M, Bany-Mohammed F, et al. Pilot feasibility study to detect mesenchymal stem cell biomarkers of bronchopulmonary dysplasia in the tracheal aspirate fluid of preterm infants. Journal of Neonatal-Perinatal Medicine 2018; 11 (1) DOI 10.3233/NPM-181722.

[2] Lal CVand Ambalavanan N. Biomarkers Early Diagnosis, and Clinical Predictors of Bronchopulmonary DysplasiaClin Perinatol. 2015; 42 (4): 739-54. doi: 10.1016/j.clp. 2015.08.004. Epub 2015 Oct 1.

[3] Nickerson BG. Bronchopulmonary dysplasia. Chronic pulmonary disease following neonatal respiratory failure. Chest 1985 Apr; 87 (4): 528-35.

[4] Jobe AH, Bancalari E. Bronchopulmonary dysplasia. Am J Respir Crit Care Med 2001; 163 (7): 1723-9.

[5] Ehrenkranz RA, Walsh MC, Vohr BR, et al. Validation of the National Institutes of Health consensus definition of bronchopulmonary dysplasia. Pediatrics 2005; 116 (6): 135360 .

[6] Bancalari E, Claure N, Sosenko IR. Bronchopulmonary dysplasia: Changes in pathogenesis, epidemiology and definition. Semin Neonatol 2003; 8 (1): 63-71.

[7] Le Cras TD, Kim DH, Markham NE, Abman AS. Early abnormalities of pulmonary vascular development in the Fawn-Hooded rat raised at Denver's altitude. Am J Physiol Lung Cell Mol Physiol 2000; 279 (2): L283-91.

[8] Anderson PJ, Doyle LW. Neurodevelopmental outcome of bronchopulmonary dysplasia. Semin Perinatol 2006; 30 (4): 227-32.

[9] Majnemer A, Riley P, Shevell M, Birnbaum R, Greenstone H,
Coates AL. Severe bronchopulmonary dysplasia increases risk for later neurological and motor sequelae in preterm survivors. Dev Med Child Neurol 2000; 42 (1): 53-60.

[10] Kinsella JP, Greenough A, Abman SH. Bronchopulmonary dysplasia. Lancet 2006; 367 (9520): 1421-31.

[11] Baveja R, Christou H. Pharmacological strategies in the prevention and management of bronchopulmonary dysplasia. Semin Perinatol 2006; 30 (4): 209-18.

[12] Todd DA, Earl M, Lloyd J, Greenberg M, John E. Cytological changes in endotracheal aspirates associated with chronic lung disease. Early Human Development 1998 51: 13-22.

[13] Merritt TA, Stuard D, Puccia J, et al. Newborn tracheal aspirate cytology: classification during respiratory distress syndrome and bronchopulmonary dysplasia. J Pediatr 198 1; 98: 949-56.

[14] Ambalavanan N and Novak ZE. Peptide Growth Factors in Tracheal Aspirates of Mechanically Ventilated Preterm Neonates. PEDIATRIC RESEARCH 2003; 53 (2): 240-244.

[15] Merritt TA, Puccia JM, Stuard D. Cytologic evaluation of pulmonary effluent in neonates with respiratory distress syndrome and bronchopulmonary dysplasia. Acta Cytol 198 1; 25: 63 1-9.

[16] D 'Ablang G. Bernard B, Zaharov 1, et al. Neonatal pulmonary cytology and bronchopulmonary dysplasia. Acta Cytol 1973; 19: 21-7.

[17] Jacobson W, Morley CJ, South M. Microscopic observations on tracheal aspirates from ventilated neonates II. The onset of bronchopulmonary dysplasia and other changes. Eur J Pediatr 1992; 151: 204-7.

[18] Doshi N, Kanbour A, Fujikura T, Klionsky B. Tracheal aspiration cytology with respiratory distress: histopathologic correlation. Acta Cytol 1982; 26: 15-21.

[19] Noack G, Mortensson W, Robertson B, Nilsson R. Correlations between radiological and cytological findings in early development of bronchopulmonary dysplasia. Eur $\mathrm{J}$ Pediatr 1993; 152: 1024-9.

[20] Rothberg AD, Miot A, Leiman G. Tracheal Aspirate Cytology and Bronchopulmonary Dysplasia. Diagnostic Cytopathology 1986; 2 (3): 212-216.

[21] Zhekova N, Perenovska P, Kalaĭdzhieva M, Pramatarova T. [Diagnostic importance of the cytologic examination of tracheal lavage fluid in high-risk newborn infants]. Akush Ginekol (Sofiia). 2007; 46 Suppl 1: 9-13. 